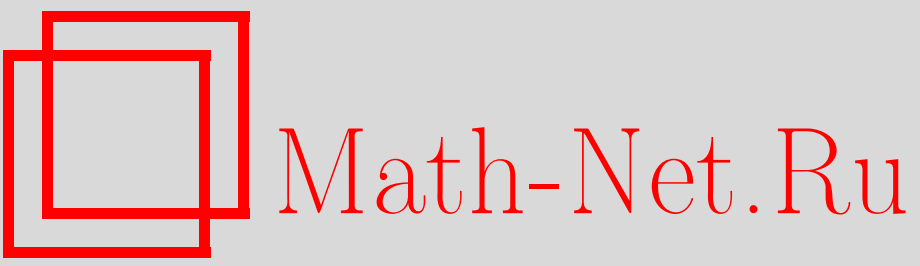

М. В. Житлухин, А. А. Муравлёв, О задаче Чернова проверки гипотез о значении сноса броуновского движения, Теория вероятн. и ее примен., 2012, том 57, выпуск 4, 778-788

DOI: https://doi.org/10.4213/tvp4480

Использование Общероссийского математического портала Math-Net.Ru подразумевает, что вы прочитали и согласны с пользовательским соглашением

http: //www . mathnet.ru/rus/agreement

Параметры загрузки:

IP : 3.80 .181 .102

26 апреля 2023 г., 14:35:44 
(c) $2012 \Gamma$

ЖИТЛУХИН М. В.*, МУРАВЛЁВ А. А.*

\title{
О ЗАДАЧЕ ЧЕРНОВА ПРОВЕРКИ ГИПОТЕЗ О ЗНАЧЕНИИ СНОСА БРОУНОВСКОГО ДВИЖЕНИЯ ${ }^{1)}$
}

\begin{abstract}
Работа содержит подробное изложение результатов, представленных в кратком сообщении [9]. Рассматривается задача Чернова последовательной проверки гипотез о положительности или отрицательности сноса броуновского движения в предположении, что он имеет нормальное распределение. Выводится интегральное уравнение, характеризующее оптимальное решающее правило, и численно находится его решение. Полученный результат дополняет результаты работ [1]-[4].
\end{abstract}

Ключевые слова и фразы: задача Чернова, последовательная проверка гипотез, задачи об оптимальной остановке, интегральные уравнения.

1. Введение. В настоящей работе мы рассмотрим задачу последовательной проверки гипотез о положительности или отрицательности сноса броуновского движения в предположении, что он имеет нормальное распределение. Данная задача была сформулирована и изучена Г. Черновым и Дж. Бриквелом в работах [1]-[4].

Пусть на вероятностном пространстве $(\Omega, \mathscr{F}, \mathbf{P})$ заданы стандартное броуновское движение $B=\left(B_{t}\right)_{t \geqslant 0}$ и нормальная случайная величина $\mu \sim \mathscr{N}\left(\mu_{0}, \sigma_{0}^{2}\right)$ с известными параметрами $\mu_{0} \in \mathbf{R}$ и $\sigma_{0}>0$, независимая от $B$. Предполагается, что $\mu$ непосредственно не наблюдаема, а наблюдению подлежит процесс $X=\left(X_{t}\right)_{t \geqslant 0}$ :

$$
X_{t}=\mu t+B_{t}, \quad t \geqslant 0 .
$$

Рассматриваемая задача заключается в проверке гипотез $H_{1}: \mu>0$ и $H_{2}: \mu \leqslant 0$ по результатам последовательного наблюдения за $X$.

Каждая последовательная процедура проверки гипотез $H_{1}$ и $H_{2}$ формально задается решаюшим правилом $(\tau, d)$, состоящим из момента остановки $\tau$, согласованного с фильтрацией $\left(\mathscr{F}_{t}\right)_{t \geqslant 0}, \mathscr{F}_{t}=\sigma\left(X_{s} ; s \leqslant t\right)$, и $\mathscr{F}_{\tau}$-измеримой функции $d$, принимающей значения \pm 1 . Момент $\tau$ является моментом прекращения наблюдения, а значение $d$ соответствует принимаемой гипотезе в момент $\tau$.

Согласно критерию Г. Чернова, решающее правило считается оптимальньлм, если оно минимизирует среднюю величину штрафа, определяемую по формуле

$$
R(\tau, d)=\mathbf{E}[c \tau+k|\mu| \mathbf{I}\{d \neq \operatorname{sgn}(\mu)\}],
$$

где $c, k>0-$ фиксированные константы и $\operatorname{sgn}(0)=-1$. Первое слагаемое представляет собой плату за наблюдение (пропорциональную времени наблюдения), а второе слагаемое - штраф за неверное решение (пропорциональный абсолютному значению $\mu$ ).

В работах [2], [3] была исследована асимптотика оптимального решающего правила при $\sigma_{0} \rightarrow 0$ и $\sigma_{0} \rightarrow \infty$. Однако точное решающие правило найдено не было. В настоящей работе мы докажем, что в оптимальном решающем правиле $\left(\tau^{*}, d^{*}\right)$

* Математический институт им. В. А. Стеклова РАН, ул. Губкина, 8, 119991 Москва, Россия; e-mail: mikhailzh@mi.ras.ru; almurav@mi.ras.ru

1) Работа выполнена при поддержке Лаборатории структурных методов анализа данных в предсказательном моделировании МФТИ (грант правительства РФ, дог. 11.G34.31.0073), РФФИ (грант № 12-01-31449-мол_а) и Министерства образования и науки РФ (дог. 14.740.11.1144). 
момент $\tau^{*}$ является моментом первого выхода некоторого случайного процесса (получающегося простым преобразованием из $X$ ) на определенную границу, а значение функции $d^{*}$ определяется по значению $X_{\tau^{*}}$. Будет получено интегральное уравнение, характеризующее оптимальную границу остановки, что даст возможность найти оптимальное решающее правило численно.

\section{2. Основная теорема.}

2.1. Далее без ограничения общности будем считать, что $c=k=1$ (иначе можно сделать замену координат; см. [1]). Зафиксируем параметры $\left(\mu_{0}, \sigma_{0}^{2}\right)$ и введем процесс $W=\left(W_{t}\right)_{0 \leqslant t \leqslant 1}$ формулой

$$
W_{t}=\sigma_{0}(1-t) X_{t /\left(\sigma_{0}^{2}(1-t)\right)}-t \frac{\mu_{0}}{\sigma_{0}}
$$

$\left(W_{1}\right.$ определяется как предел $W_{t}$ при $\left.t \rightarrow 1\right)$. Несложно проверить, что $W$ является непрерывным гауссовским процессом с независимыми приращениями, $\mathbf{E} W_{t}=0$, $\mathbf{E} W_{t}^{2}=t$ и $W_{0}=0$. Таким образом, $W$ является стандартным броуновским движением (винеровским процессом).

Мы покажем, что оптимальное решающее правило в задаче различения гипотез $H_{1}$ и $H_{2}$ может быть найдено путем решения задачи об оптимальной остановке для процесса $W$ (см. теорему ниже):

$$
V_{\mu_{0}, \sigma_{0}}=\inf _{\tau \leqslant 1} \mathbf{E}\left[\frac{2}{\sigma_{0}^{3}(1-\tau)}-\left|W_{\tau}+\frac{\mu_{0}}{\sigma_{0}}\right|\right],
$$

где инфимум берется по всем моментам остановки $\tau \leqslant 1$ относительно фильтрации $\left(\mathscr{F}_{t}^{W}\right)_{t \leqslant 1}, \mathscr{F}_{t}^{W}=\sigma\left(W_{s} ; s \leqslant t\right)$. Под решением понимается момент остановки, на котором достигается инфимум.

Для краткости дальнейших обозначений введем функцию

$$
G(t, x)=\frac{1}{\sqrt{t}} \varphi\left(\frac{x}{\sqrt{t}}\right)-\frac{|x|}{t} \Phi\left(-\frac{x}{\sqrt{t}}\right), \quad t>0, \quad x \in \mathbf{R},
$$

где $\varphi(x)=e^{-x^{2} / 2} / \sqrt{2 \pi}$ и $\Phi(x)=\int_{-\infty}^{x} \varphi(t) d t$ - стандартные нормальные плотность и функция распределения.

Теорема 1. 1) Пусть момент остановки $\tau_{W}^{*}$ является решением задачи (2). Тогда оптимальное решающее правило $\left(\tau^{*}, d^{*}\right)$ в задаче различения гипотез $H_{1}$ и $\mathrm{H}_{2}$ имеет вид

$$
\tau^{*}=\frac{\tau_{W}^{*}}{\sigma_{0}^{2}\left(1-\tau_{W}^{*}\right)}, \quad d^{*}=\operatorname{sgn}\left(X_{\tau^{*}}+\frac{\mu_{0}}{\sigma_{0}^{2}}\right) .
$$

2) Момент $\tau_{W}^{*}=\tau_{W}^{*}\left(\mu_{0}, \sigma_{0}\right)$ имеет вид

$$
\tau_{W}^{*}\left(\mu_{0}, \sigma_{0}\right)=\inf \left\{0 \leqslant t \leqslant 1:\left|W_{t}+\frac{\mu_{0}}{\sigma_{0}}\right| \geqslant a_{\sigma_{0}}(t)\right\},
$$

где $a_{\sigma_{0}}(t):[0,1] \rightarrow \mathbf{R}_{+}-$невозрастаюшая функция на $[0,1]$, являющаяся единственным решением интегрального уравнения

$$
\frac{G(1-t, a(t))}{1-t}=\int_{t}^{1} \frac{2}{\sigma_{0}^{3}(1-s)^{2}}\left[\Phi\left(\frac{a(s)-a(t)}{\sqrt{s-t}}\right)-\Phi\left(\frac{-a(s)-a(t)}{\sqrt{s-t}}\right)\right] d s
$$

в классе непрерывных функиий $а(t)$, удовлетворяющих условиям

$$
a(t)>0 \quad \text { npu } t<1, \quad a(1)=0
$$


и условию

$$
\int_{t}^{1} \frac{1}{(1-s)^{2}}\left[\Phi\left(\frac{a(s)-x}{\sqrt{s-t}}\right)-\Phi\left(\frac{-a(s)-x}{\sqrt{s-t}}\right)\right] d s<\infty
$$

$\partial л я$ всех $t \in[0,1), x \in \mathbf{R}$.

2.2. Отметим, что в работе [1] задача различения $H_{1}$ и $H_{2}$ сводится к задаче об оптимальной остановке для другого процесса. А именно, вводится процесс $X^{\prime}=\left(X_{t}^{\prime}\right)_{t \geqslant 1 / \sigma_{0}^{2}}, X_{t}^{\prime}=X_{t-1 / \sigma_{0}^{2}}+\mu_{0} / \sigma_{0}^{2}$, который удовлетворяет стохастическому дифференциальному уравнению

$$
d X_{t}^{\prime}=\frac{X_{t}^{\prime}}{t} d t+d \bar{B}_{t}^{\prime}, \quad t \geqslant \frac{1}{\sigma_{0}^{2}}
$$

с некоторым броуновским движением $\bar{B}^{\prime}$.

В работе [1] доказывается, что оптимальное решающее правило может быть найдено посредством решения задачи об оптимальной остановке

$$
V^{\prime}(t, x)=\inf _{\tau \geqslant t} \mathbf{E}_{t, x}\left[\tau-G\left(\tau, X_{\tau}^{\prime}\right)\right], \quad t=\frac{1}{\sigma_{0}^{2}}, \quad x=\frac{\mu}{\sigma_{0}^{2}}
$$

где $\mathbf{E}_{t, x}$ обозначает математическое ожидание в предположении, что процесс $X^{\prime}=$ $\left(X_{s}^{\prime}\right)_{s \geqslant t}$ задается уравнением (8) с начальным условием $X_{t}^{\prime}=x$; инфимум берется по всем моментам остановки $\tau \geqslant t$ относительно фильтрации $\left(\mathscr{F}_{s}^{X^{\prime}}\right)_{s \geqslant t}, \mathscr{F}_{s}^{X^{\prime}}=\sigma\left(X_{u}^{\prime} ; t \leqslant\right.$ $u \leqslant s)$. Если $\tau^{*}$ - оптимальный момент остановки в задаче (9) для $t=1 / \sigma_{0}^{2}, x=\mu / \sigma_{0}^{2}$, то $\left(\tau^{*}-1 / \sigma_{0}^{2}, \operatorname{sgn}\left(X_{\tau^{*}}^{\prime}\right)\right)$ является оптимальным решающим правилом для данных значений $\mu_{0}$ и $\sigma_{0}^{2}$.

Далее в [1] устанавливается, что оптимальный момент остановки в (9) имеет вид

$$
\tau^{*}(t, x)=\inf \left\{s \geqslant t:\left|X_{s}^{\prime}\right| \geqslant b(s)\right\}
$$

где $b(s): \mathbf{R}_{+} \rightarrow \mathbf{R}_{+}$- некоторая функция, строго положительная при $t>0$ (оптимальная граница остановки). Отметим, что эта функция не зависит от параметров $\mu_{0}, \sigma_{0}$.

Как непосредственно следует из конструкции процессов $W$ и $X^{\prime}$, оптимальные границы остановки в задачах (2) и (9) связаны равенством

$$
b(t)=\sigma_{0} t \cdot a_{\sigma_{0}}\left(1-\frac{1}{\sigma_{0}^{2} t}\right), \quad t \geqslant \frac{1}{\sigma_{0}^{2}} .
$$

Таким образом, зная семейство $\left\{a_{\sigma_{0}}(\cdot)\right\}_{\sigma_{0}>0}$, можно найти $b(\cdot)$, и наоборот.

В работах [2], [3] была исследована асимптотика границы остановки $b(t)$ при $t \rightarrow \infty$ и $t \rightarrow 0$ (что эквивалентно $\sigma_{0} \rightarrow 0$ и $\sigma_{0} \rightarrow \infty$ ). С помощью же теоремы, доказываемой в настоящей работе, можно получить (по крайней мере численно) явный вид границ остановки. Таким образом, настоящая работа дополняет уже имеющиеся в литературе результаты.

2.3. Для численного нахождения решения уравнения (5) можно воспользоваться «методом обратной индукции». Пусть $0 \leqslant t_{0}<t_{1}<\cdots<t_{n}=1-$ разбиение отрезка $[0,1]$. Согласно (6), имеем $a\left(t_{n}\right)=0$. Зная значения $a\left(t_{k}\right), a\left(t_{k+1}\right), \ldots, a\left(t_{n}\right)$ и приближенно вычисляя интеграл (5) для $t=t_{k-1}$ по значениям подынтегральной функции в точках $t_{k-1}, t_{k}, \ldots, t_{n}$, получим уравнение, из которого находится $a\left(t_{k-1}\right)$. Повторяя данную процедуру, можно найти все значения $a\left(t_{0}\right), \ldots, a\left(t_{n}\right)$.

В качестве примера на рис. 1 слева приведен график оптимальной границы остановки $\alpha_{\sigma_{0}}(t)$ для процесса $W$ при $\sigma_{0}=\sqrt{2}$; справа приведен график оптимальной границы $b(t)$ для процесса $X^{\prime}$. 

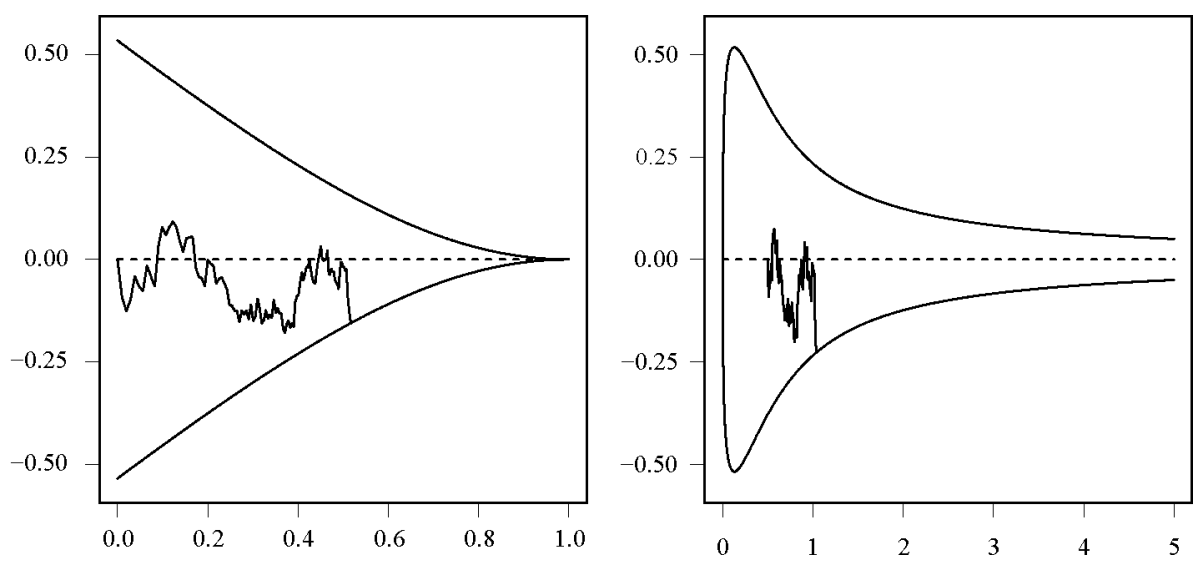

Рис. 1. Слева: оптимальная границы остановки $a_{\sigma_{0}}(t)$; справа: оптимальная граница остановки $b(t)$.

\section{3. Доказательство теоремы.}

3.1. Для доказательства теоремы воспользуемся стандартной схемой, используемой для подобных утверждений (см., например, [7]). Приводимое здесь доказательство во многом повторяет аналогичные рассуждения работы [10].

Сразу отметим, что, очевидно, оптимальное решающее правило следует искать среди правил $(\tau, d)$, для которых $\mathbf{E} \tau<\infty$. Поэтому далее будем рассматривать только такие решающие правила.

Для любого решающего правила $(\tau, d)$ справедливо равенство

$$
R(\tau, d)=\mathbf{E}\left[\tau+\mathbf{E}\left(\mu^{-} \mid \mathscr{F}_{\tau}\right) \mathbf{I}\{d=+1\}+\mathbf{E}\left(\mu^{+} \mid \mathscr{F}_{\tau}\right) \mathbf{I}\{d=-1\}\right],
$$

где $\mu^{+}=\max \{\mu, 0\}, \mu^{-}=\max \{-\mu, 0\}$. Отсюда ясно, что для нахождения оптимального решающего правила $\left(\tau^{*}, d^{*}\right)$ достаточно найти момент остановки $\tau^{*}$, минимизирующий (в классе моментов остановки, согласованных с фильтрацией $\left.\left(\mathscr{F}_{t}\right)_{t \geqslant 0}\right)$ математическое ожидание

$$
\mathscr{E}(\tau)=\mathbf{E}\left[\tau+\min \left\{\mathbf{E}\left(\mu^{-} \mid \mathscr{F}_{\tau}\right), \mathbf{E}\left(\mu^{+} \mid \mathscr{F}_{\tau}\right)\right\}\right]
$$

и задать функцию $d^{*}$ формулой

$$
d^{*}= \begin{cases}+1, & \mathbf{E}\left(\mu^{-} \mid \mathscr{F}_{\tau^{*}}\right) \leqslant \mathbf{E}\left(\mu^{+} \mid \mathscr{F}_{\tau^{*}}\right) \\ -1, & \mathbf{E}\left(\mu^{-} \mid \mathscr{F}_{\tau^{*}}\right)>\mathbf{E}\left(\mu^{+} \mid \mathscr{F}_{\tau^{*}}\right)\end{cases}
$$

Воспользовавшись нормальностью условного распределения $\mu$ :

$$
\operatorname{Law}\left(\mu \mid \mathscr{F}_{t}\right)=\mathscr{N}\left(\frac{X_{t}+\mu_{0} / \sigma_{0}^{2}}{t+1 / \sigma_{0}^{2}}, \frac{1}{t+1 / \sigma_{0}^{2}}\right)
$$

(см. [1]), получаем, что для любого момента остановки $\tau$

$$
\mathscr{E}(\tau)=\mathbf{E}\left[\tau+G\left(\tau+\frac{1}{\sigma_{0}^{2}}, X_{\tau}+\frac{\mu_{0}}{\sigma_{0}^{2}}\right)\right],
$$

где функция $G(t, x)$ задается формулой (3). 
Из обновляющего представления для процесса $X$ (см., например, $[11, \S 7.4])$ следует, что процесс $\bar{B}_{t}=X_{t}-\int_{0}^{t} \mathbf{E}\left(\mu \mid \mathscr{F}_{s}\right) d s$ является стандартным броуновским движением $\left(\bar{B}^{\prime}\right.$ из формулы (8) связано с $\bar{B}$ соотношением $\left.\bar{B}_{t}^{\prime}=\bar{B}_{t-1 / \sigma_{0}^{2}}\right)$. Используя тот факт, что $\mathbf{E}\left(\mu \mid \mathscr{F}_{t}\right)=\left(X_{t}+\mu_{0} / \sigma_{0}^{2}\right) /\left(t+1 / \sigma_{0}^{2}\right)$ согласно (11), находим, что $X$ удовлетворяет уравнению

$$
d X_{t}=\frac{X_{t}+\mu_{0} / \sigma_{0}^{2}}{t+1 / \sigma_{0}^{2}} d t+d \bar{B}_{t}
$$

Заметим, что функция $G(t, x)+|x| /(2 t)$ принадлежит классу $\mathscr{C}^{1,2}((0, \infty) \times \mathbf{R})$. C помощью непосредственного применения формулы Ито убеждаемся в справедливости выражения

$$
\begin{aligned}
G\left(t+\frac{1}{\sigma_{0}^{2}}, X_{t}+\frac{\mu_{0}}{\sigma_{0}^{2}}\right)= & G\left(\frac{1}{\sigma_{0}^{2}}, \frac{\mu_{0}}{\sigma_{0}^{2}}\right)+\frac{\left|\mu_{0}\right|}{2}-\frac{\left|X_{t}+\mu_{0} / \sigma_{0}^{2}\right|}{2\left(t+1 / \sigma_{0}^{2}\right)} \\
& +\int_{0}^{t} \frac{2 \Phi\left(\left|X_{s}+\mu_{0} / \sigma_{0}^{2}\right| / \sqrt{s+1 / \sigma_{0}^{2}}\right)-1}{2\left(s+1 / \sigma_{0}^{2}\right)} d \bar{B}_{s}
\end{aligned}
$$

Тогда для любого момента остановки $\tau$ такого, что $\mathbf{E} \tau<\infty$, имеем

$$
\mathscr{E}(\tau)=\mathbf{E}\left[\tau-\frac{\left|X_{\tau}+\mu_{0} / \sigma_{0}^{2}\right|}{2\left(\tau+1 / \sigma_{0}^{2}\right)}\right]+G\left(\frac{1}{\sigma_{0}^{2}}, \frac{\mu_{0}}{\sigma_{0}^{2}}\right)+\frac{\left|\mu_{0}\right|}{2},
$$

где мы воспользовались тем, что подынтегральная функция стохастического интеграла в формуле (12) ограничена и, следовательно, его математическое ожидание равно 0, так как $\mathbf{E} \tau<\infty$.

Непосредственно проверяется, что процесс $M=\left(M_{t}\right)_{t \geqslant 0}$, задаваемый формулой

$$
M_{t}=\frac{X_{t}+\mu_{0} / \sigma_{0}^{2}}{\sigma_{0}\left(t+1 / \sigma_{0}^{2}\right)}-\frac{\mu_{0}}{\sigma_{0}}
$$

является мартингалом с нулевым средним, и с помощью замены времени его можно преобразовать к броуновскому движению. А именно, процесс $W=\left(W_{t}\right)_{t \leqslant 1}$,

$$
W_{t}=M_{t /\left(\sigma_{0}^{2}(1-t)\right)}
$$

является стандартным броуновским движением (данная формула связана с (1) простым преобразованием). Тогда

$$
\mathscr{E}(\tau)=\frac{\sigma_{0}}{2} \mathbf{E}\left[\frac{2}{\sigma_{0}^{3}\left(1-\tau_{W}\right)}-\left|W_{\tau_{W}}+\frac{\mu_{0}}{\sigma_{0}}\right|\right]+G\left(\frac{1}{\sigma_{0}^{2}}, \frac{\mu_{0}}{\sigma_{0}^{2}}\right)+\frac{\left|\mu_{0}\right|}{2}-\frac{1}{\sigma_{0}^{2}},
$$

где $\tau_{W}$ - момент остановки относительно фильтрации $\left(\mathscr{F}_{t}^{W}\right)_{t \leqslant 1}, \mathscr{F}_{t}^{W}=\sigma\left(W_{s} ; s \leqslant t\right)$, задаваемый формулой

$$
\tau_{W}=\frac{\sigma_{0}^{2} \tau}{1+\sigma_{0}^{2} \tau} .
$$

Таким образом, для нахождения оптимального решающего правила $\left(\tau^{*}, d^{*}\right)$ в задаче различения гипотез $H_{1}$ и $H_{2}$ достаточно найти решение $\tau_{W}^{*}$ задачи об оптимальной остановке (2) и определить $\tau^{*}$ и $d^{*}$ по формулам (4) (выражение для $\tau^{*}$ следует непосредственно из (13), а выражение для $d^{*}$ следует из (10) после вычисления условных математических ожиданий). 
3.2. Чтобы найти решение задачи (2), положим для краткости $c=2 / \sigma_{0}^{3}$ и введем функцию цены, позволяя двумерному процессу $\left(t, W_{t}\right)$ начинаться из любой точки $(t, x) \in[0,1] \times \mathbf{R}:$

$$
V(t, x)=\inf _{\tau_{W} \leqslant 1-t} \mathbf{E}\left[\frac{c}{1-\left(\tau_{W}+t\right)}-\left|W_{\tau_{W}}+x\right|\right]-\frac{c}{1-t},
$$

где мы полагаем $V(1, x)=0$ для всех $x$. Ясно, что $V_{\mu_{0}, \sigma_{0}}=V\left(0, \mu_{0} / \sigma_{0}\right)+c$.

Далее для краткости будем вместо $\tau_{W}$ писать просто $\tau$, поскольку это не приведет к недоразумению.

C помощью общего метода теории оптимальной остановки (см., например, [7, гл. I]) устанавливается, что фазовое пространство $[0,1] \times \mathbf{R}$ разбивается на множество остановки $D$ и множество продолжения наблюдения $C$ :

$$
D=\{(t, x): V(t, x)=-|x|\}, \quad C=\{(t, x): V(t, x)<-|x|\}
$$

(здесь $-|x|$ является выигрышем от мгновенной остановки). При этом инфимум в (14) достигается на моменте первого попадания рассматриваемого процесса в множество $D$ :

$$
\tau^{*}(t, x)=\inf \left\{s \leqslant 1-t:\left(s+t, W_{s}+x\right) \in D\right\} .
$$

Ясно, что каждое из множеств $D$ и $C$ симметрично относительно прямой $x=0$. Доказывается, что функция $V(t, x)$ непрерывна (см. п. 4.1), и, следовательно, множество $D$ замкнуто.

Несложно заметить, что функция $V(t, x)+|x|$ не убывает по $t$ и $x \geqslant 0$, так как для любого момента остановки $\tau$

$$
\begin{aligned}
\frac{c}{1-(\tau+t)}-\frac{c}{1-t} & \leqslant \frac{c}{1-\left(\tau+t^{\prime}\right)}-\frac{c}{1-t^{\prime}} \quad \text { для любых } t \leqslant t^{\prime}, \\
|x|-\left|W_{\tau}+x\right| & \leqslant\left|x^{\prime}\right|-\left|W_{\tau}+x^{\prime}\right| \quad \text { для любых } 0 \leqslant x \leqslant x^{\prime},
\end{aligned}
$$

причем если $t \leqslant t^{\prime}$, то инфимум в (14) для $t$ берется по большему классу моментов остановки, чем для $t^{\prime}$.

Кроме того, $V(t, 0)<0$ для всех $t<1$, так как $\mathbf{E}\left|W_{s}\right| \sim \sqrt{s}$ и, следовательно, в (14) можно положить $\tau_{W}=\tau \equiv s$ для такого малого $s$, что $\mathbf{E}\left|W_{s}\right|>c(1-(s+t))^{-1}$ $c(1-t)^{-1}$.

Отсюда следует, что множество $C$ представимо в виде

$$
C=\{(t, x): t \in[0,1],|x|<a(t)\}
$$

где $a(t):[0,1] \rightarrow \mathbf{R}_{+}$- некоторая невозрастающая функция на $[0,1]$ и $a(t)>0$ при $t<1$ (отметим, что функция $a(t)$ зависит от $c$, или, что эквивалентно, от $\sigma$ ).

Покажем, что $a(t)$ непрерывна на $[0,1]$. Так как $a(t)$ не возрастает и множество остановки $D$ замкнуто, то $a(t)$ непрерывна справа. Докажем непрерывность слева. Заметим, что, поскольку $\mathbf{E} W_{\tau^{*}}=0$, справедлива формула

$$
V(t, x)=\mathbf{E}\left[\frac{c}{1-\left(\tau^{*}+t\right)}+W_{\tau^{*}}-\left|W_{\tau^{*}}+x\right|\right]-\frac{c}{1-t},
$$

где $\tau^{*}=\tau^{*}(t, x)$.

Предположим, что $a(t-)>a(t)$ для некоторого $t \in[0,1]$. Рассмотрим значения функции (15) в точках $(t-\varepsilon, x)$ для $x=(a(t-)+a(t)) / 2$ и достаточно малых $\varepsilon>0$. 
Обозначим $A_{\varepsilon}=\left\{\omega: W_{s}(\omega)+x \in[a(t), a(t-)], s \leqslant \varepsilon\right\}$ и $r_{\varepsilon}=c(1-t)^{-1}-c(1-(t-\varepsilon))^{-1}$. Тогда имеем

$$
\begin{aligned}
V(t-\varepsilon, x) & \stackrel{(\text { a) }}{\geqslant} \mathbf{E}\left[\left(r_{\varepsilon}-x\right) \mathbf{I}\left(A_{\varepsilon}\right)\right]+\mathbf{E}\left[\left(W_{\tau^{*}}-\left|W_{\tau^{*}}+x\right|\right) \mathbf{I}\left(\Omega \backslash A_{\varepsilon}\right)\right] \\
& \stackrel{(\text { b) }}{\geqslant} r_{\varepsilon}-x-\mathbf{P}\left(\Omega \backslash A_{\varepsilon}\right)\left(r_{\varepsilon}-x+\sqrt{\mathbf{E}\left(W_{\tau^{*}}-\left|W_{\tau^{*}}+x\right|\right)^{2}}\right) .
\end{aligned}
$$

В (а) для того, чтобы получить первое слагаемое, мы воспользовались тем, что $\tau^{*}(\omega)=t$ и $W_{\tau^{*}}-\left|W_{\tau^{*}}+x\right|=-x$ на множестве $A_{\varepsilon}$, а чтобы получить второе неравенством $c\left(1-\left(\tau^{*}+t\right)\right)^{-1}-c(1-t)^{-1} \geqslant 0$. В (b) мы воспользовались неравенством Коши-Буняковского-Шварца для $\mathbf{E}\left[\left(W_{\tau^{*}}-\left|W_{\tau^{*}}+x\right|\right) \mathbf{I}\left(\Omega \backslash A_{\varepsilon}\right)\right]$.

Согласно неравенству Дуба,

$$
\mathbf{P}\left(\Omega \backslash A_{\varepsilon}\right) \leqslant 2 \exp \left(-\frac{(a(t-)-a(t))^{2}}{8 \varepsilon}\right) .
$$

Так как $r_{\varepsilon}=O(\varepsilon)$ при $\varepsilon \rightarrow 0$, то найдется такое достаточно малое $\varepsilon>0$, что $V(t-$ $\varepsilon, x)>-x$, что противоречит тому, что $(t-\varepsilon, x) \in C$. Это доказывает непрерывность границы $a(t)$.

С помощью аналогичных рассуждений доказывается, что $a(1)=0$. Отсюда и из непрерывности следует, в частности, что $a(t)$ конечно для всех $t \in[0,1]$.

Покажем, как получить уравнение (5). Пусть $\mathscr{L}$ обозначает инфинитезимальный оператор двумерного процесса $\left(t, W_{t}\right)$ :

$$
\mathscr{L}=\frac{\partial}{\partial t}+\frac{1}{2} \frac{\partial^{2}}{\partial x^{2}}
$$

Следуя общим методам теории оптимальной остановки (см. $[7, \S \S 7-9])$, доказываем, что

$$
\begin{array}{rlrl}
V(t, x) & \in \mathscr{C}^{1,2}(C) \cup \mathscr{C}^{1,2}\left(D^{0}\right), & \\
\mathscr{L} V(t, x) & =-c(1-t)^{-2}, & & (t, x) \in C, \\
V(t, x) & =-|x|, & & (t, x) \in D, \\
V_{x+}^{\prime}(t, \pm a(t)) & =V_{x-}^{\prime}(t, \pm a(t)), & & t \in[0,1],
\end{array}
$$

где $D^{0}$ обозначает внутренность множества $D$.

Свойства (16)-(18) следуют из марковского характера задачи (см. [7, §7]) и определения множества $D$. Свойство (19) является принципом гладкого склеивания (см. п. 4.2), из него следует равенство $V_{x}^{\prime}(t, \pm a(t)) \equiv \mp 1$, поскольку $V_{x \pm}^{\prime}(t, \pm a(t))=$ $G_{x}^{\prime}(t, \pm a(t))=\mp 1$. Кроме того, так как математическое ожидание $\mathbf{E}[\cdot]$ под знаком inf в (14) есть вогнутая функция от $x$, то

$$
\text { функция } x \mapsto V(t, x) \text { вогнута, } t \in[0,1] \text {. }
$$

Итак, в рассматриваемой задаче выполнены следующие свойства: (а) функция $V(t, x)$ входит в $\mathscr{C}^{1,2}(C) \cup \mathscr{C}^{1,2}\left(D^{0}\right)$ и непрерывна на $[0,1] \times \mathbf{R}_{+}$, функция $a(t)$ непрерывна и имеет ограниченную вариацию; (b) $\mathscr{L} V(t, x)$ локально ограничено на $C$ и на $D^{0}$ (следует из $\left.(17)-(18)\right) ;($ c) $x \mapsto V(t, x)$ является вогнутой функцией для каждого $t \in[0,1] ;(\mathrm{d}) V_{x}^{\prime}(t, \pm a(t))=\mp 1$ для $t \in[0,1]$. Это позволяет применить к $V(t, x)$ формулу Ито с локальным временем на кривых [5]:

$$
\mathbf{E} V\left(1, W_{1-t}+x\right)=V(t, x)+\mathbf{E} \int_{t}^{1} \mathscr{L} V\left(s, W_{s-t}+x\right) \mathbf{I}\left\{W_{s-t}+x \neq \pm a(s)\right\} d s
$$




$$
\begin{aligned}
& +\mathbf{E} \int_{t}^{1} V_{x}^{\prime}\left(s, W_{s-t}+x\right) \mathbf{I}\left\{W_{s-t}+x \neq \pm a(s)\right\} d W_{s-t} \\
& +\frac{1}{2} \mathbf{E} \int_{t}^{1} \Delta V_{x}^{\prime}(s, a(s)) \mathbf{I}\left\{W_{s-t}+x=a(s)\right\} d L_{s-t}^{a(\cdot)} \\
& +\frac{1}{2} \mathbf{E} \int_{t}^{1} \Delta V_{x}^{\prime}(s,-a(s)) \mathbf{I}\left\{W_{s-t}+x=-a(s)\right\} d L_{s-t}^{-a(\cdot)}
\end{aligned}
$$

где $L^{ \pm a(\cdot)}$ - процессы локального времени процесса $W$ на кривых $\pm a(s)$ (см. подробнее [5]).

В силу определения функции $V(t, x)$ имеем $V\left(1, W_{1-t}+x\right)=-\left|W_{1-t}+x\right|$. Из принципа гладкого склеивания (19) следует, что два последних слагаемых в (20) равны нулю. Также заметим, что производная $V_{x}^{\prime}(t, x)$ равномерно ограничена, так как для любых $x_{1} \leqslant x_{2}$ и $t \in[0,1]$ выполнены неравенства $x_{1}-x_{2} \leqslant V\left(t, x_{2}\right)-V\left(t, x_{1}\right) \leqslant x_{2}-x_{1}$. Действительно, в силу четности функции $V(t, x)$ по $x$ достаточно проверить данное утверждение для $0 \leqslant x_{1} \leqslant x_{2}$. Тогда левое неравенство следует из того, что, как было отмечено выше, функция $V(t, x)+|x|$ не убывает при $x \geqslant 0$, а правое неравенство следует из соотношения

$$
V\left(t, x_{2}\right)-V\left(t, x_{1}\right) \leqslant \sup _{\tau \leqslant 1-t} \mathbf{E}\left[\left|W_{\tau}+x_{1}\right|-\left|W_{\tau}+x_{2}\right|\right] \leqslant x_{2}-x_{1} .
$$

Из ограниченности $V_{x}^{\prime}(t, x)$ вытекает равенство нулю математического ожидания стохастического интеграла в (20).

Используя (17) и равенство $\mathscr{L} V(t, x)=0$ при $(t, x) \in D^{0}$ (которое справедливо, поскольку $V(t, x)=|x|$ на $D)$, получаем

$$
V(t, x)=-\mathbf{E}\left|W_{1-t}+x\right|+\int_{t}^{1} \frac{c}{(1-s)^{2}} \mathbf{P}\left(\left|W_{s-t}+x\right|<a(s)\right) d s .
$$

Беря $x=a(t)$ и используя равенство $V(t, a(t))=-a(t)$ (которое справедливо, поскольку $(t, a(t)) \in D)$, получаем уравнение (5) после непосредственного вычисления математического ожидания и вероятности, входящих в правую часть.

3.3. Докажем единственность решения уравнения (5), применяя метод, предложенный в [6]. Пусть $a(t)$ - решение этого уравнения, являющееся оптимальной границей остановки, а $\bar{a}(t)$ - некоторое другое решение, являющееся непрерывной функцией, удовлетворяющей (6) и (7). Рассмотрим функцию

$$
\bar{V}(t, x)=-\mathbf{E}\left|W_{1-t}+x\right|+\int_{t}^{1} \frac{c}{(1-s)^{2}} \mathbf{P}\left(\left|W_{s-t}+x\right|<\bar{a}(s)\right) d s .
$$

Заметим, что $\bar{V}(t, \bar{a}(t))=-\bar{a}(t)$ в силу того, что $\bar{a}(t)$ удовлетворяет (5).

Пользуясь строго марковским свойством броуновского движения, можно проверить, что для любого момента остановки $\tau \leqslant 1-t$ справедливо равенство

$$
\bar{V}(t, x)=\mathbf{E} \bar{V}\left(\tau+t, W_{\tau}+x\right)+\mathbf{E} \int_{t}^{\tau+t} \frac{c}{(1-s)^{2}} \mathbf{I}\left(\left|W_{s-t}+x\right|<\bar{a}(s)\right) d s .
$$

Для фиксированных $(t, x)$ рассмотрим момент $\tau_{\bar{a}}=\inf \left\{s \geqslant 0:\left|W_{s}+x\right|=\bar{a}(s+t)\right\}$. Учитывая, что $\mathbf{I}\left(\left|W_{s-t}+x\right|<\bar{a}(s)\right)=0$ при $t \leqslant s \leqslant \tau_{\bar{a}}+t$, если $|x| \geqslant \bar{a}(t)$, и $\mathbf{I}\left(\mid W_{s-t}+\right.$ $x \mid<\bar{a}(s))=1$ при $t \leqslant s<\tau_{\bar{a}}+t$, если $|x|<\bar{a}(t)$, находим

$$
\begin{array}{ll}
\bar{V}(t, x)=-\mathbf{E}\left|W_{\tau_{\bar{a}}}+x\right|=-|x|, \quad|x| \geqslant \bar{a}(t), \\
\bar{V}(t, x)=\mathbf{E}\left[\frac{c}{1-\left(\tau_{\bar{a}}+t\right)}-\frac{c}{1-t}-\left|W_{\tau_{\bar{a}}}+x\right|\right], \quad|x|<\bar{a}(t) .
\end{array}
$$


Во втором равенстве первой формулы мы воспользовались тем, что при $x \geqslant \bar{a}(t)$ имеем $\left|W_{\tau_{\bar{a}}}+x\right|=\bar{a}\left(\tau_{\bar{a}}\right) \geqslant 0$ и, следовательно, $\mathbf{E}\left|W_{\tau_{\bar{a}}}+x\right|=\mathbf{E} W_{\tau_{\bar{a}}}+x=x$. Аналогично, $\mathbf{E}\left|W_{\tau_{\bar{a}}}+x\right|=-x$ при $x \leqslant-\bar{a}(t)$. Во второй формуле мы воспользовались тем, что $\int_{t}^{\tau_{\bar{a}}+t^{2}} c(1-s)^{-2} d s=c\left(1-\left(\tau_{\bar{a}}+t\right)\right)^{-1}-c(1-t)^{-1}$. Из этих формул следует, что $\bar{V}(t, x) \geqslant V(t, x)$ для всех $t \in[0,1], x \in \mathbf{R}$.

Предположим, что $\bar{a}(t)>a(t)$ для некоторого $t \in[0,1)$. Возьмем $x=\bar{a}(t)$ и рассмотрим момент остановки $\tau_{a}=\inf \left\{s \geqslant 0:\left|W_{s}+x\right|=a(t+s)\right\}$. Тогда из (21), используя соотношения $\bar{V}(t, x)=-x$ и $\mathbf{E} \bar{V}\left(t+\tau_{a}, W_{\tau_{a}}+x\right) \geqslant \mathbf{E} V\left(t+\tau_{a}, W_{\tau_{a}}+x\right)=$ $-\mathbf{E}\left(W_{\tau_{a}}+x\right)=-x$, получаем

$$
-x \geqslant-x+\mathbf{E} \int_{t}^{\tau_{a}+t} \frac{c}{(1-s)^{2}} \mathbf{I}\left(\left|W_{s-t}+x\right|<\bar{a}(s)\right) d s .
$$

Однако математическое ожидание в правой части строго положительно, так как $W_{s-t}+x$ мгновенно попадает в область ниже кривой $\bar{a}(s)$. Полученное противоречие означает, что $\bar{a}(t) \leqslant a(t)$ для всех $t \in[0,1]$.

Предположим, $\bar{a}(t)<a(t)$ для некоторого $t \in[0,1)$ и возьмем $x=\bar{a}(t)$. Тогда $\bar{V}(t, x)=-x$ и $\bar{V}\left(t+\tau_{a}, W_{\tau_{a}}+x\right)=-\left|W_{\tau_{a}}+x\right|$, так как $a\left(t+\tau_{a}\right) \geqslant \bar{a}\left(t+\tau_{a}\right)$ по доказанному выше. Из формулы (21) следует, что

$$
-x \leqslant-\mathbf{E}\left|W_{\tau_{a}}+x\right|+\mathbf{E} \int_{t}^{\tau_{a}+t} \frac{c}{(1-s)^{2}} d s=V(t, x) .
$$

Таким образом, $V(t, x) \geqslant-x$, что противоречит $(t, x) \in C$. Значит, $\bar{a}(t) \geqslant a(t)$, что доказывает равенство $\bar{a}(t)=a(t)$ для всех $t \in[0,1]$.

Теорема доказана.

\section{4. Приложение.}

4.1. Докажем непрерывность функции цены $V(t, x)$. Так как $V(t, x)$ не убывает по $t$ и $x \geqslant 0$, то непрерывность достаточно доказать лишь по каждой переменной. Непрерывность по $x$ очевидна, так как для любых $t \in[0,1), x, x^{\prime} \in \mathbf{R}$, справедливо неравенство

$$
\left|V\left(t, x^{\prime}\right)-V(t, x)\right| \leqslant \sup _{\tau \leqslant 1-t} \mathbf{E}|| W_{\tau}+x|-| W_{\tau}+x^{\prime}|| \leqslant\left|x^{\prime}-x\right| .
$$

Для доказательства непрерывности по $t$ зафиксируем $x \in \mathbf{R}$ и рассмотрим произвольные $0 \leqslant t<1,0 \leqslant \varepsilon<1-t$. Справедлива оценка

$$
\begin{aligned}
\mathbf{E}\left[\frac{c}{1-(\tau+t)}-\left|W_{\tau}+x\right|\right]-\frac{c}{1-t} \\
\quad \geqslant \mathbf{E}\left[\frac{c}{1-(\tau \vee \varepsilon+t)}-\left|W_{\tau \vee \varepsilon}+x\right|+\left|W_{\varepsilon}+x\right|-\left|W_{\tau \wedge \varepsilon}+x\right|\right]-\frac{c}{1-(\varepsilon+t)} \\
\quad \geqslant \mathbf{E} V\left(t+\varepsilon, W_{\varepsilon}+x\right)+\mathbf{E}\left|W_{\varepsilon}+x\right|-\mathbf{E}\left|W_{\tau \wedge \varepsilon}+x\right|,
\end{aligned}
$$

где первое неравенство следует из непосредственных алгебраических преобразований, а во втором неравенстве мы воспользовались марковским свойством броуновского движения. Далее, имеем

$$
|\mathbf{E}| W_{\varepsilon}+x|-\mathbf{E}| W_{\tau \wedge \varepsilon}+x|| \leqslant \mathbf{E}\left|W_{\varepsilon}-W_{\tau \wedge \varepsilon}\right|=\mathbf{E}\left|\widetilde{W}_{\varepsilon-\tau \wedge \varepsilon}\right| \leqslant \sqrt{2 \varepsilon},
$$

где процесс $\widetilde{W}=\left(\widetilde{W}_{t}\right)_{t \leqslant \varepsilon}, \widetilde{W}_{t}=W_{\varepsilon}-W_{\varepsilon-t}$, является броуновским движением. Доказательство правого неравенства в (23) можно найти, например, в [8]. Беря в (22) 
инфимум по всем моментам $\tau \leqslant 1-t$, получаем, что

$$
V(t, x) \geqslant \mathbf{E} V\left(t+\varepsilon, W_{\varepsilon}+x\right)-\sqrt{2 \varepsilon} .
$$

По доказанному выше, $\left|\mathbf{E} V\left(t+\varepsilon, W_{\varepsilon}+x\right)-V(t+\varepsilon, x)\right| \leqslant \mathbf{E}\left|W_{\varepsilon}\right|=\varepsilon \sqrt{2 / \pi}$. Таким образом,

$$
0 \geqslant V(t, x)-V(t+\varepsilon, x) \geqslant-\left(\sqrt{2 \varepsilon}+\varepsilon \sqrt{\frac{2}{\pi}}\right),
$$

что доказывает непрерывность функции $V(t, x)$ по $t$.

4.2. Докажем принцип гладкого склеивания (19). Заметим, что левая и правая производные $V_{x-}^{\prime}(t, a(t))$ и $V_{x+}^{\prime}(t, a(t))$ существуют в силу вогнутости функции $V(t, x)$ по $x$, причем $V_{x+}^{\prime}(t, a(t))=-1$, так как $V(t, x)=-x$ при $x \geqslant a(t)$ (точки из множества остановки).

Рассмотрим левую производную. Зафиксируем $t \in[0,1)$ и возьмем $x=a(t)$. Для достаточно малых $\varepsilon>0$ справедливо неравенство

$$
\frac{V(t, x-\varepsilon)-V(t, x)}{-\varepsilon}=\frac{V(t, x-\varepsilon)+x}{-\varepsilon} \geqslant \frac{\varepsilon-x+x}{-\varepsilon}=-1,
$$

где в левом равенстве мы воспользовались тем, что $V(t, x)=-|x|$, а в правом - тем, что $V(t, x-\varepsilon) \leqslant-|x-\varepsilon|$. Следовательно,

$$
V_{x-}^{\prime}(t, x) \geqslant-1
$$

Докажем противоположное неравенство. Выберем малое $\varepsilon>0$, и пусть $\tau^{*}-$ оптимальный момент остановки для $V(t, x-\varepsilon)$, т.е. $\tau^{*}=\inf \left\{s \geqslant 0:\left|W_{s}+x-\varepsilon\right| \geqslant\right.$ $a(t+s)\}$. Тогда

$$
\frac{V(t, x-\varepsilon)-V(t, x)}{-\varepsilon} \leqslant \frac{\mathbf{E}\left|W_{\tau^{*}}+x\right|-\mathbf{E}\left|W_{\tau^{*}}+x-\varepsilon\right|}{-\varepsilon}
$$

где мы воспользовались тем, что

$$
\begin{gathered}
V(t, x) \leqslant \mathbf{E}\left[c\left(1-\left(\tau^{*}+t\right)\right)^{-1}-\left|B_{\tau^{*}}+x\right|\right]-c(1-t)^{-1}, \\
V(t, x-\varepsilon)=\mathbf{E}\left[c\left(1-\left(\tau^{*}+t\right)\right)^{-1}-\left|B_{\tau^{*}}+x-\varepsilon\right|\right]-c(1-t)^{-1} .
\end{gathered}
$$

Преобразовывая получившееся выражение, имеем

$$
\begin{aligned}
& \mathbf{E}\left|W_{\tau^{*}}+x\right|-\mathbf{E}\left|W_{\tau^{*}}+x-\varepsilon\right|=\varepsilon \mathbf{P}\left(W_{\tau^{*}}+x-\varepsilon=a(t+\tau)\right) \\
& \quad+\mathbf{E}\left[\left(\left|W_{\tau^{*}}+x\right|-\left|W_{\tau^{*}}+x-\varepsilon\right|\right) \mathbf{I}\left(W_{\tau^{*}}+x-\varepsilon=-a(t+\tau)\right)\right] .
\end{aligned}
$$

Второе слагаемое по модулю оценивается величиной $\varepsilon \mathbf{P}\left(W_{\tau^{*}}+x-\varepsilon=-a(t+\tau)\right)=o(\varepsilon)$. Тогда

$$
\lim _{\varepsilon \downarrow 0} \frac{V(t, x-\varepsilon)-V(t, x)}{-\varepsilon} \leqslant-1,
$$

что доказывает неравенство $V_{x-}^{\prime}(t, x) \leqslant-1$, откуда следует справедливость принципа гладкого склеивания.

Авторы выражают благодарность А.Н. Ширяеву, обратившему их внимание на рассматриваемую задачу. 


\title{
СПИСОК ЛИТЕРАТУРЫ
}

1. Chernoff $H$. Sequential tests for the mean of a normal distribution. - Proceedings of the Fourth Berkeley Symposium on Mathematical Statistics and Probability, v. 1. Berkeley: Univ. of California Press, 1961, p. 79-91.

2. Breakwell J., Chernoff $H$. Sequential tests for the mean of a normal distribution II (large $t$ ). - Ann. Math. Statist., 1964, v. 35, № 1, p. 162-173.

3. Chernoff $H$. Sequential tests for the mean of a normal distribution III (small $t$ ). Ann. Math. Statist., 1965, v. 36, № 1, p. 28-54.

4. Chernoff $H$. Sequential tests for the mean of a normal distribution IV (discrete case). Ann. Math. Statist., 1965, v. 36, № 1, p. 55-68.

5. Peskir G. A change-of-variable formula with local time on curves. - J. Theoret. Probab., 2005, v. 18, p. 499-535.

6. Peskir G. On the American option problem. - Math. Finance, 2005, v. 15, p. 169-181.

7. Peskir G., Shiryaev A. Optimal Stopping and Free-Boundary Problems. Birkhäuser, 2006, $500 \mathrm{p}$

8. Дубинс Л.Е., Шепn Л. А., Ширяев А.Н. Оптимальные правила остановки и максимальные неравенства для процессов Бесселя. - Теория вероятн. и ее примен., 1993, т. 38, в. 2 , с. $288-330$.

9. Житлухин М. В., Муравлёв A.А. Об уравнениях для оптимальных границ в задаче Чернова различения двух гипотез. - Успехи матем. наук, 2011, т. 66, № 5, c. $183-184$.

10. Житлухин М. В., Ширяев А. Н. Байесовские задачи о разладке на фильтрованных вероятностных пространствах. - Теория вероятн. и ее примен., 2012, т. 57, в. 3, c. $453-470$.

11. Липцер Р. Ш., Ширяев А.Н. Статистика случайных процессов. М.: Наука, 1974, $696 \mathrm{c}$

\section{МНОГОМЕРНЫЕ ЭКСТРЕМУМЫ СЛУЧАЙНЫХ ПРИЗНАКОВ ЧАСТИЦ В НАДКРИТИЧЕСКИХ ВЕТВЯЩИХСЯ ПРОЦЕССАХ ${ }^{1)}$}

\begin{abstract}
Рассматриваются надкритические ветвящиеся процессы с дискретным временем, в которых каждая частица обладает несколькими случайными признаками. Нас интересуют максимумы этих признаков в популяции. Рассмотрены два случая: регулярный (когда число непосредственных потомков имеет конечные первый и второй моменты, а совместное распределение признаков принадлежит области притяжения некоторого многомерного экстремального закона), а затем более общий. Описаны классы невырожденных предельных законов для многомерных экстремумов при линейной нормировке. Особое внимание уделено структурам зависимости, описываемым копулами. Для них получено функциональное уравнение и доказана теорема о продолжении. Установлена связь с макс-полуустойчивыми распределениями.
\end{abstract}

* Московский государственный университет им. М. В. Ломоносова, механико-математический факультет, кафедра теории вероятностей, Москва, Россия; e-mail: alebedev@mech.math.msu.su

1) Работа выполнена при поддержке по грантам РФФИ 07-01-00077, 07-01-00373, 11-01-00050. 